\title{
Analysis of gene expression profile identifies potential biomarkers for atherosclerosis
}

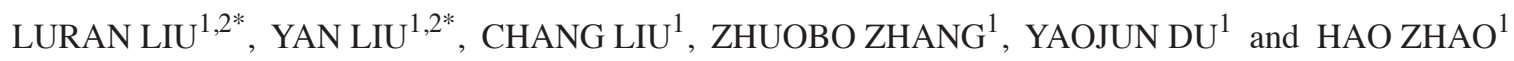 \\ Departments of ${ }^{1}$ Neurology and ${ }^{2}$ Urology, The Fourth Affiliated Hospital of Harbin Medical University, \\ Harbin, Heilongjiang 150001, P.R. China
}

Received February 28, 2015; Accepted December 30, 2015

DOI: $10.3892 / \mathrm{mmr} .2016 .5650$

\begin{abstract}
The present study aimed to identify potential biomarkers for atherosclerosis via analysis of gene expression profiles. The microarray dataset no. GSE20129 was downloaded from the Gene Expression Omnibus database. A total of 118 samples from the peripheral blood of female patients was used, including 47 atherosclerotic and 71 non-atherosclerotic patients. The differentially expressed genes (DEGs) in the atherosclerosis samples were identified using the Limma package. Gene ontology term and Kyoto Encyclopedia of Genes and Genomes pathway analyses for DEGs were performed using the Database for Annotation, Visualization and Integrated Discovery tool. The recursive feature elimination (RFE) algorithm was applied for feature selection via iterative classification, and support vector machine classifier was used for the validation of prediction accuracy. A total of 430 DEGs in the atherosclerosis samples were identified, including 149 up- and 281 downregulated genes. Subsequently, the RFE algorithm was used to identify 11 biomarkers, whose receiver operating characteristic curves had an area under curve of 0.92 , indicating that the identified 11 biomarkers were representative. The present study indicated that APH1B, JAM3, FBLN2, CSAD and PSTPIP2 may have important roles in the progression of atherosclerosis in females and may be potential biomarkers for early diagnosis and prognosis as well as treatment targets for this disease.
\end{abstract}

\section{Introduction}

Atherosclerosis is a chronic inflammatory disease which mainly results from the abnormal accumulation of

Correspondence to: Dr Luran Liu, Department of Neurology, The Fourth Affiliated Hospital of Harbin Medical University, 37 Yiyuan Street, Nangang, Harbin, Heilongjiang 150001, P.R. China

E-mail: luranliulrl@163.com

\section{${ }^{*}$ Contributed equally}

Key words: atherosclerosis, recursive feature elimination, support vector machine classifier, biomarker macrophages, white blood cells and lipids in the artery wall, and eventually form mature plaques (1). It causes various types of cardiovascular disease, which account for $\sim 50 \%$ of all mortalities worldwide (2). To date, the underlying molecular pathogenesis of atherosclerosis has remained largely elusive. An understanding of the molecular basis of atherosclerotic processes is crucial for the development of potential therapeutic strategies.

Atherosclerosis is characterized by endothelial dysfunction, vascular inflammation, plaque formation and diminished oxygen supply to target organs $(3,4)$. Endothelial dysfunction is an initial step in atherosclerosis. Dysfunctional endothelial cells release a large variety of pro-inflammatory mediators, leading to the amplification of inflammatory response $(5,6)$, and systemic inflammation may in turn contribute to endothelial dysfunction and accelerated atherosclerosis $(7,8)$. Considering the crucial roles of inflammation in the pathogenesis of atherosclerosis, systemic markers of inflammation may be the most obvious candidates for potential biomarkers for early diagnosis, risk prediction and clinical prognosis of this disease (9). C-reactive protein has been implicated in multiple aspects of atherosclerosis and is currently the best validated inflammatory biomarker to predict the risk of atherosclerotic events $(10,11)$. Fibrinogen, apolipoproteins and interleukins are associated with the progression of atherosclerosis and have a great relevance in risk prediction (12). Inflammatory molecules, including intercellular adhesion molecule-1 and vascular cell adhesion molecule-1, are also considered as biomarkers for risk prediction of atherosclerosis $(11,13)$. Several studies have identified a large number of biomarkers with potential prognostic value for atherosclerosis; their overall clinical predictive value, however, is modest (14). Therefore, it is urgently required to identify a reliable, specific and cost-effective biomarker for facilitating the clinical diagnosis of atherosclerosis.

The microarray dataset no. GSE20129 from the Gene Expression Omnibus database (GEO; http://www.ncbi.nlm. nih.gov/geo/) has been utilized to reveal variations in gene expression associated with coronary artery calcification between Caucasian and African Americans based on a multiethnic study of atherosclerosis (15); furthermore, the dataset has been used to reveal systemic transcriptional alterations of innate and adaptive immune signaling pathways in atherosclerosis (16). The present study used the microarray 
dataset GSE20129 to identify differentially expressed genes (DEGs) associated with atherosclerosis using comprehensive bioinformatics methods. In addition, the recursive feature elimination (RFE) algorithm was applied for feature selection via iterative classification, and the support vector machine (SVM) classifier was used for the validation of prediction accuracy. The present study aimed to identify potential biomarkers for atherosclerosis via analysis of gene expression profiles.

\section{Materials and methods}

Microarray data. The array dataset GSE20129, which was provided by Huang et al (15), was downloaded from the GEO database. A total of 118 samples from the peripheral blood of females, including 47 atherosclerotic and 71 non-atherosclerotic patients, was used for expression profiling. The raw CEL data and annotation files were obtained based on the Illumina human Ref-8 v2.0 expression beadchip platform (Illumina Inc., San Diego, CA, USA) for subsequent analyses.

Data processing and screening of DEGs. All probe IDs of the dataset were transformed into gene symbols based on probe annotation files. If multiple probes corresponded to the same gene symbol, the mean value was calculated as the expression value of this gene. Z-score normalization of expression values was preformed and then the expression value $(\mathrm{X})$ of each sample was corrected. The corrected expression value $\left(\mathrm{X}^{\prime}\right)$ was calculated as follows: $\mathrm{X}^{\prime}=(\mathrm{X}$-mean $) /$ standard deviation.

The DEGs in samples from atherosclerotic patients compared with those in non-atherosclerotic patients were screened using the Limma package (http://www.bioconductor.org/packages/release/bioc/html/ limma.html) (17) in $\mathrm{R} . \mathrm{P}<0.01$ was defined as the cut-off value for $\mathrm{DEG}$ screening.

Unsupervised hierarchical clustering analysis. Unsupervised hierarchical clustering analysis (18) for DEGs was performed to effectively distinguish atherosclerotic and non-atherosclerotic samples using cluster 3.0 (19) and to visualize these samples using the heatmap tool in TreeView (20). Filtering and normalization of all the expression data were performed using cluster 3.0. Genes with expression in at least $80 \%$ of all samples were retained, while others were discarded. All of the genes and samples were median centered (21) and normalized. The similarity matrixes were correlation centered (22) and normalized.

Functional enrichment analyses. The Gene Ontology (GO) tool (23) provides functional annotations for large-scale genomic or transcription data. The Kyoto Encyclopedia of Genes and Genomes database (KEGG; http://www. genome.ad.jp/kegg/) (24) holds information on pathways and networks of molecules or genes. The Database for Annotation, Visualization and Integrated Discovery (DAVID; http://david.abcc.ncifcrf.gov/) software (25) is an integrated biological database and analytic tool for systematic annotation of biological meaning for large lists of genes or proteins.
To understand the biological significance of DEGs, GO term and KEGG pathway analyses for up-and downregulated DEGs were performed using the DAVID online tool. The significance threshold was set at $\mathrm{P}<0.05$.

Feature selection with the RFE algorithm. Feature selection offers classification models with high relevance by eliminating irrelevant features (26). In order to obtain candidates for diagnostic and prognostic biomarkers of atherosclerosis, the RFE algorithm $(27,28)$ in python was applied for feature selection from the constellation of DEGs via iterative classification. Classification analyses were performed using a linear Support Vector Classification (SVC; linear kernel, step $=1$; cross-validation $=5$ ) provided by the python module scikit-learn (29). This recursive procedure adopted a backward elimination strategy to iteratively remove irrelevant features and was iterated until all relevant features were obtained.

Prediction with SVM classifier. All of the relevant features were processed with a linear SVM classifier (30) for classification prediction, which was repeated five times. Linear kernel and five-fold cross-validation of all samples were performed in this prediction procedure to assess classification performance. To further illustrate the prediction accuracy of this method, a receiver operating characteristic curve (ROC) was implemented and the area under curve (AUC) was calculated.

\section{Results}

DEG screening and hierarchical clustering analysis. Based on probe annotation files, a total of 18,196 gene symbols were acquired after transformation. A total of 430 DEGs with $\mathrm{P}<0.01$ were screened using the Limma package, including 149 upregulated and 281 downregulated genes. The results of the unsupervised hierarchical clustering analysis were visualized in a heatmap (Fig. 1).

Functional enrichment analyses. Functional enrichment analyses for DEGs were performed using DAVID, and the significantly enriched GO terms and KEGG pathways are shown in Tables I and II, respectively. According to the enrichment results, the over-represented GO terms of upregulated genes were mainly associated with ribonucleoprotein complex biogenesis, ribosome biogenesis and positive regulation of T-cell proliferation, while downregulated genes were mainly associated with response to lipopolysaccharides, immune response and responses to molecules of bacterial origin (Table I). In addition, upregulated genes were significantly enriched in viral myocarditis and purine metabolism, while downregulated genes were significantly involved in leukocyte transendothelial migration and the Notch signaling pathway (Table II).

Feature selection with the RFE algorithm and prediction with SVM classifier. By applying the RFE algorithm, a total of 11 biomarkers were obtained, including APH1B, JAM3, FBLN2, CSAD and PSTPIP2 (Table III). These 11 biomarkers were then processed with the SVM classifier for classification prediction. The AUC of the ROC obtained 

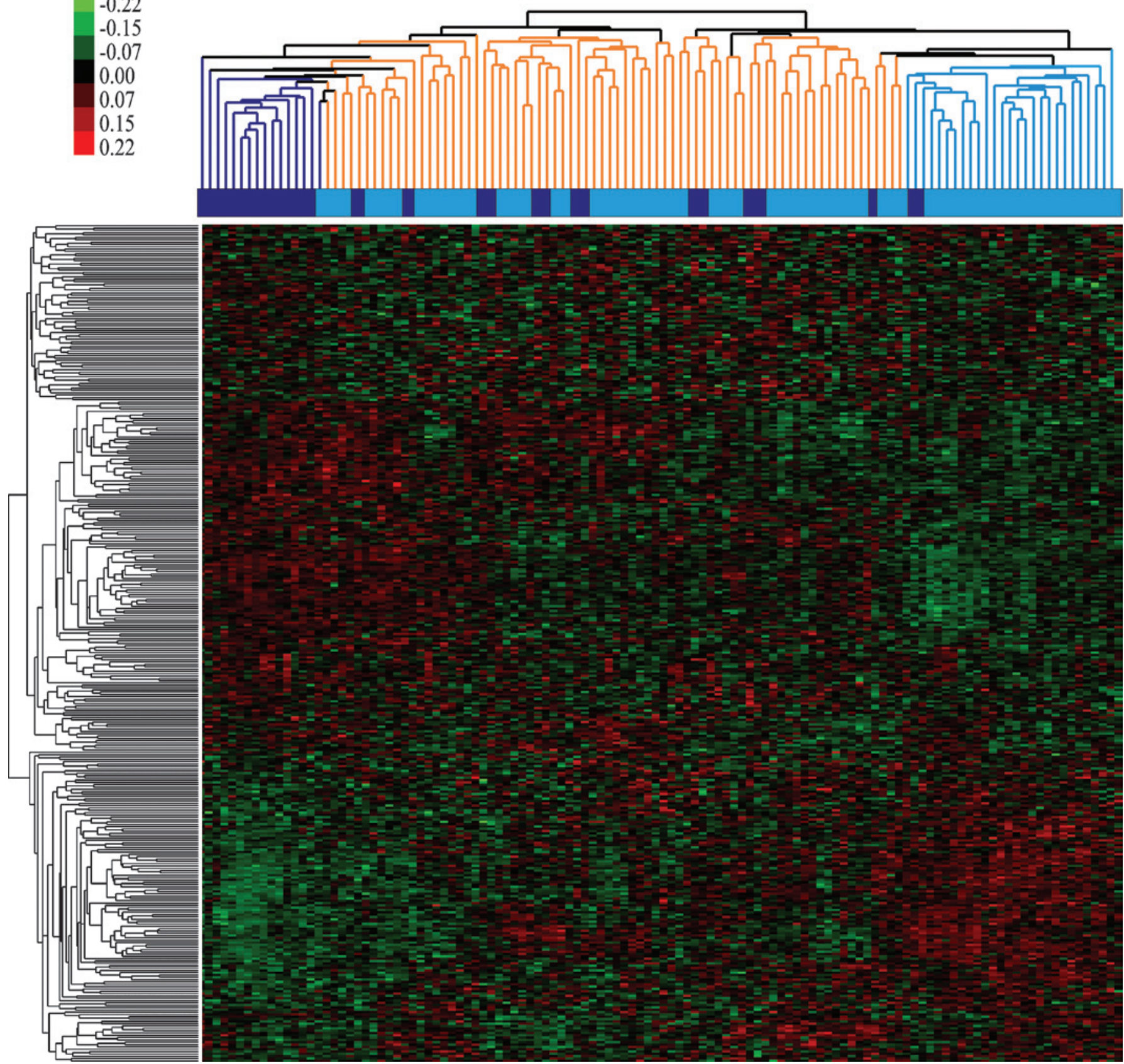

Figure 1. Heat map of hierarchical clustering analysis. The abscissa represents clustering of specimens with atherosclerosis samples in dark blue and non-atherosclerosis samples in light blue, and the mixture of the two sample groups in orange. The ordinate represents differentially expressed genes and the clustering of genes. Red indicates upregulated genes while green signifies downregulated genes.

with the SVM classifier was calculated as 0.92 (Fig. 2), indicating that the identified 11 biomarkers were representative. In order to verify that the biomarkers that we screened were representative for the DEGs in atherosclerotic patients, all 430 DEGs were processed with the SVM classifier, resulting in an AUC of the ROC of 0.95 (Fig. 3), which confirmed that the 11 biomarkers were representative as the prediction accuracy of the identified 11 biomarkers was markedly higher than the randomly selected 11 DEGs. In addition, to rule out any randomization, the 11 DEGs were randomly selected as features which were subjected to the process of classification for 100,000 times, and the AUC of every step was calculated. The results showed that the rank position of the AUC corresponding to 11 biomarkers screened was 33 ( $\mathrm{P}=0.0033)$, suggesting that the prediction accuracy of the screened biomarkers was significantly higher than that of random results.

\section{Discussion}

Atherosclerosis is the proximate cause of occlusive arterial disease (31). Identification of potential biomarkers that have crucial roles in the progression of atherosclerosis is important for the development of therapeutic approaches. In the present study, a bioinformatics approach was used to predict potential biomarkers for the diagnosis and treatment 
Table I. Over-represented GO terms enriched by differentially expressed genes.

\begin{tabular}{lrr}
\hline Term & Count & P-value \\
\hline Upregulated genes & 6 & 0.010748 \\
GO:0022613 - Ribonucleoprotein complex biogenesis & 5 & 0.012705 \\
GO:0042254 - Ribosome biogenesis & 3 & 0.033574 \\
GO:0042102 - Positive regulation of T-cell proliferation & 8 & 0.042602 \\
GO:0007010 - Cytoskeletal organization & 3 & 0.045367 \\
GO:0006941 - Striated muscle contraction & & 7 \\
Downregulated genes & 22 & 7 \\
GO:0032496 - Response to lipopolysaccharides & 18 & $0.36 \times 10^{-4}$ \\
GO:0006955 - Immune response & 11 & 0.001271 \\
GO:0002237 - Response to molecules of bacterial origin & 4 & 0.002148 \\
GO:0009611 - Response to wounding & 5 & 0.002768 \\
GO:0002684 - Positive regulation of immune system processes & 5 \\
GO:0001892 - Embryonic placenta development & 5 & 0.005054 \\
GO:0045936 - Negative regulation of phosphate metabolic processes & 8 & 0.005334 \\
GO:0010563 - Negative regulation of phosphorus metabolic processes & 22 & 0.005334 \\
GO:0050778 - Positive regulation of immune response & 12 & 0.005621 \\
GO:0042127 - Regulation of cell proliferation & 0.005981 \\
GO:0006954 - Inflammatory response & 0.008674 \\
\hline
\end{tabular}

GO, gene ontology.

Table II. Significantly enriched Kyoto Encyclopedia of Genes and Genomes pathways among differentially expressed genes.

\begin{tabular}{lll}
\hline Term & Count & P-value \\
\hline Upregulated & 3 & \\
hsa05416: Viral myocarditis & 4 & 0.02366 \\
hsa00230: Purine metabolism & & \\
Downregulated & 7 & 0.01940 \\
hsa04670: Leukocyte transendothelial & & \\
migration & 4 & 0.04248 \\
hsa04330: Notch signaling pathway & & \\
\hline hsa, Homo sapiens. & & \\
\hline
\end{tabular}

of atherosclerosis. The screening of gene expression profiles of atherosclerotic patients identified 11 biomarkers, including APH1B, JAM3, FBLN2, CSAD and PSTPIP2, and the AUC of the ROC obtained using SVM classifier was calculated as 0.92 , which indicated that these 11 biomarkers were representative. The crucial roles of these genes in the pathogenesis of atherosclerosis and their potential diagnostic and therapeutic values for this disease warrant further evaluation.

APH1B is a functional component of the gamma-secretase enzyme complex, which catalyzes the cleavage of transmembrane proteins such as Notch receptors and amyloid-b precursor protein (APP) (32). Notch activation induces senescence of endothelial cells and results in pro-inflammatory responses, and
Table III. Biomarkers screened using the recursive feature elimination algorithm.

\begin{tabular}{llr}
\hline Biomarker & P-value & logFC \\
\hline APH1B & $5.81 \times 10^{-4}$ & -0.089736 \\
HIATL1 & $1.44 \times 10^{-3}$ & -0.111004 \\
JAM3 & $2.24 \times 10^{-3}$ & 0.164411 \\
HOXB13 & $2.25 \times 10^{-3}$ & -0.031921 \\
FBLN2 & $2.35 \times 10^{-3}$ & 0.049115 \\
RNF148 & $2.82 \times 10^{-3}$ & -0.030481 \\
RALB & $2.84 \times 10^{-3}$ & -0.093036 \\
CPEB3 & $3.07 \times 10^{-3}$ & -0.059267 \\
ABHD14B & $6.30 \times 10^{-3}$ & 0.069626 \\
CSAD & $7.75 \times 10^{-3}$ & -0.041617 \\
PSTPIP2 & $9.45 \times 10^{-3}$ & -0.096693 \\
\hline
\end{tabular}

FC, fold change.

Notch signaling has pivotal roles in the pathogenesis of atherosclerosis (33). Blockade of Notch ligand delta-like 4 - Notch signaling attenuates the development of atherosclerosis (34). APP has also been found to have a potential pathogenic role in carotid atherogenesis (35). In addition, the single-nucleotide polymorphism Phe217Leu (rs1047552; T>G) in the APH1B gene is associated with premature coronary atherosclerosis (36). It can therefore be speculated that APH1B may be implicated in the progression of atherosclerosis and function as a biomarker for risk prediction. 


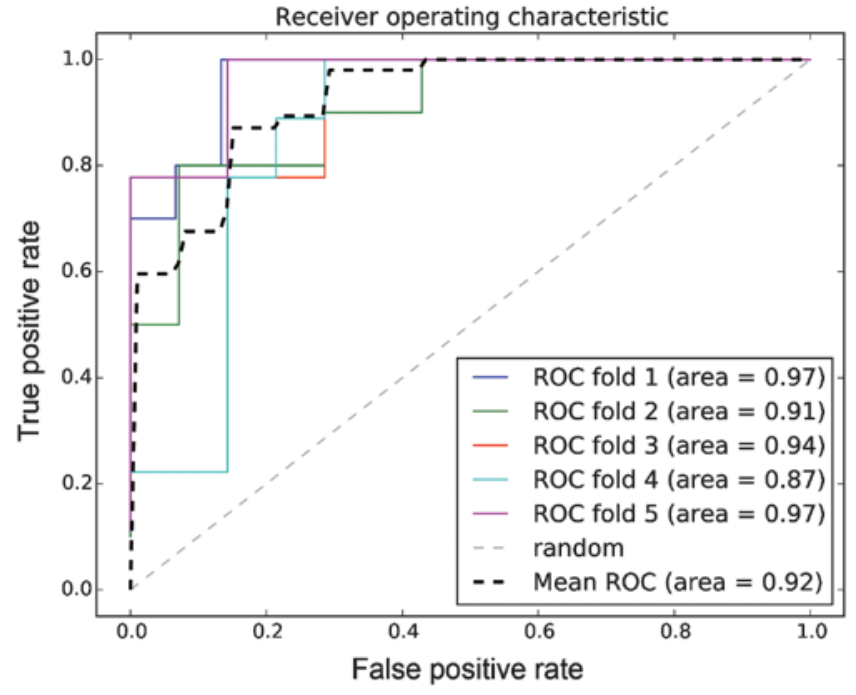

Figure 2. ROC of support vector machine classifier constructed by 11 biomarkers. Five curves represent the results of every time (refinement cycle) in five-fold cross-validation of all samples. The black dotted line represents the mean ROC, and the light gray dotted line represents the random ROC. ROC, receiver operating characteristic curve; area, area under curve.

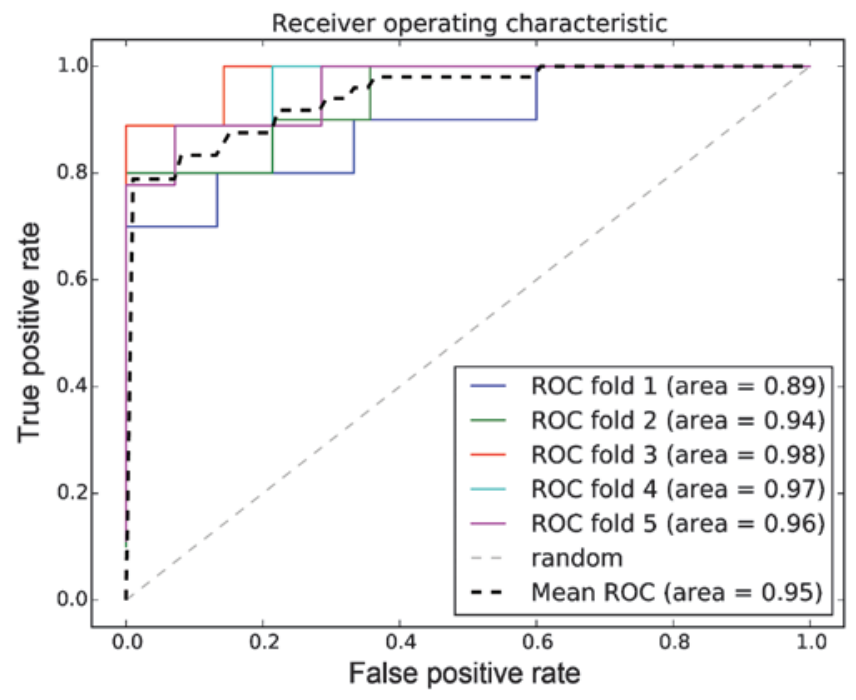

Figure 3. ROC of support vector machine classifier constructed by all differentially expressed genes. Five curves represent the results of every time (refinement cycle) in five-fold cross-validation of all samples. The black dotted line represents the mean ROC, and the light gray dotted line represents the random ROC. ROC, receiver operating characteristic curve; area, area under curve.

Junctional adhesion molecule 3 (JAM3) is a member of vascular adhesion molecules, which mediate adhesion and interactions among cells or between cells and the extracellular matrix (37). Increased expression and activation of adhesion molecules in vascular endothelial cells and circulating leukocytes stimulate leukocyte recruitment into the vascular endothelium, which is an important step in the development of atherosclerosis $(38,39)$. JAM-C has been shown to be involved in the control of inflammatory leukocyte recruitment in atherosclerosis (40). JAM3 may act as a counter-receptor for Mac-1, which mediates leukocyte-platelet interactions between vascular cells, and may thereby provide a molecular target for antagonizing interactions involved in several vascular pathologies, such as in atherothrombosis (37). In the present study, JAM3 was identified as a potential biomarker for atherosclerosis, which was in line with the abovementioned previous findings and suggested that JAM3 may be a potential therapeutic target in atherosclerosis.

The FBLN2 gene encodes an the extracellular matrix protein fibulin 2. Upregulation of FBLN2 may promote the arterial response to injury and accelerate atherogenesis among patients with diabetes (41). In addition, FBLN2 may lead to the aberrant regulation of activator protein-1 factors, which have been shown to be associated with the progression of atherosclerosis (42). Thus, FBLN2 may be implicated in the progression of atherosclerosis and serve as a biomarker for this disease.

Cysteine sulfinic acid decarboxylase (CSAD) can catalyze the conversion of cysteine sulfinic acid to hypotaurine, which may limit the generation of taurine (43). Taurine has been shown to have the potential protective effects against cardiovascular diseases and can effectively prevent the progression of atherosclerotic diseases (44). Therefore, it may be hypothesized that CSAD may have roles in the progression of atherosclerosis via limiting the generation of taurine.

Proline-serine-threonine phosphatase-interacting protein 2 (PSTPIP2) is expressed in macrophages and is tyrosine-phosphorylated in response to colony-stimulating factor-1 (CSF-1) (45). CSF-1 is thought to have a role in the accumulation of cholesterol-laden macrophages (foam cells) in atherosclerotic plaques (46). Chitu and Stanley (47) found that CSF-1 was involved in several inflammatory disorders, such as artherosclerosis. Although the causal roles of PSTPIP2 in the development of atherosclerosis have not been fully elucidated, it may be speculated that PSTPIP2 has a pivotal role in the progression of atherosclerosis via interaction with CSF-1. The results of the present study indicated that PSTPIP2 may be a potential biomarker for atherosclerosis, which requires further investigation.

Only gene expression profiles of atherosclerotic/nonatherosclerotic females were investigated in the present study. However, atherosclerosis may have different pathologies in males and females; therefore, sex differences should be explored. Furthermore, there was no experimental validation in the present study; therefore, further investigations are required in order to verify the present findings and speculation. In particular, additional prospective biomarkers, including HIATL1, HOXB13, RNF148, RALB, CPEB3 and ABHD14B, were identified, which merit further investigation and discussion.

In conclusion, the bioinformatics analysis of the present study indicated that APH1B, JAM3, FBLN2, CSAD and PSTPIP2 may have important roles in the pathogenesis of atherosclerosis. They may be considered as potential biomarkers for early diagnosis and prognosis as well as therapeutic targets for this disease, which requires experimental validation.

\section{Acknowledgements}

The present study was supported by Provincial Science and Technology Research which is named super early 
rehabilitation after acute cerebral apoplexy clinical research (grant no. GC12C306-3).

\section{References}

1. Hansson GK, Robertson AK and Söderberg-Nauclér C: Inflammation and atherosclerosis. Annu Rev Pathol 1: 297-329, 2006.

2. Cooper R, Cutler J, Desvigne-Nickens P, Fortmann SP, Friedman L, Havlik R, Hogelin G, Marler J, McGovern P, Morosco G, et al: Trends and disparities in coronary heart disease, stroke, and other cardiovascular diseases in the United States: Findings of the national conference on cardiovascular disease prevention. Circulation 102: 3137-3147, 2000.

3. Mannarino E and Pirro M: Molecular biology of atherosclerosis. Clin Cases Miner Bone Metab 5: 57-62, 2008

4. Davignon J and Ganz P: Role of endothelial dysfunction in atherosclerosis. Circulation 109 (23 Suppl 1): SIII-27-SIII-32, 2004.

5. Vanhoutte PM: Endothelial dysfunction: The first step toward coronary arteriosclerosis. Circ J 73: 595-601, 2009.

6. Mudau M, Genis A, Lochner A and Strijdom H: Endothelial dysfunction: The early predictor of atherosclerosis. Cardiovasc J Afr 23: 222-231, 2012.

7. Antoniades C,Demosthenous M, Tousoulis D, Antonopoulos AS, Vlachopoulos C, Toutouza M, Marinou K, Bakogiannis C, Mavragani K, Lazaros G, et al: Role of asymmetrical dimethylarginine in inflammation-induced endothelial dysfunction in human atherosclerosis. Hypertension 58: 93-98, 2011.

8. Sitia S, Tomasoni L, Atzeni F, Ambrosio G, Cordiano C, Catapano A, Tramontana S, Perticone F, Naccarato P Camici $\mathrm{P}$, et al: From endothelial dysfunction to atherosclerosis. Autoimmun Rev 9: 830-834, 2010.

9. Ragino YI, Chernjavski AM, Polonskaja YV, Tchimbal SY, Redjkin DA, Semaeva EV, Kashtanova EV and Voevoda MI: Blood levels of inflammatory and destructive biomarkers in coronary atherosclerosis of different severity. Bull Exp Biol Med 149: 587-590, 2010.

10. Koenig W and Khuseyinova N: Biomarkers of atherosclerotic plaque instability and rupture. Arterioscler Thromb Vasc Biol 27: 15-26, 2007.

11. Kampoli AM, Tousoulis D, Antoniades C, Siasos G and Stefanadis C: Biomarkers of premature atherosclerosis. Trends Mol Med 15: 323-332, 2009.

12. Montagnana M, Lippi G, Salvagno GL, Franchini M, Targher G and Guidi GC: Role of biochemical risk factors and markers for the risk of atherosclerosis. Recenti Prog Med 99: 215-222, 2008 (In Italian).

13. Zhu YP, Shen T, Lin YJ, et al: Astragalus polysaccharides suppress ICAM-1 and VCAM-1 expression in TNF- $\alpha$-treated human vascular endothelial cells by blocking NF- $\mathrm{kB}$ activation. Acta Pharmacol Sin 34: 1036-1042, 2013.

14. Revkin JH, Shear CL, Pouleur HG, Ryder SW and Orloff DG: Biomarkers in the prevention and treatment of atherosclerosis: Need, validation, and future. Pharmacol Rev 59: 40-53, 2007.

15. Huang CC, Lloyd-Jones DM, Guo X, Rajamannan NM, Lin S, Du P, Huang Q, Hou L and Liu K: Gene expression variation between African Americans and whites is associated with coronary artery calcification: The multiethnic study of atherosclerosis. Physiol Genomics 43: 836-843, 2011

16. Huang CC, Chantler P, VanGilder R and Barr T: Systemic transcriptional alterations of innate-adaptive immune signaling pathways in atherosclerosis, ischemic stroke and myocardial infarction. Arterioscler Thromb Vasc Biol 34: A546-A546, 2014.

17. Smyth GK: Limma: Linear models for microarray data. In: Bioinformatics and Computational Biology Solutions using $\mathrm{R}$ and Bioconductor. Gentleman R, Carey V, Dudoit S, Irizarry R and Huber W (eds). Springer, New York, pp397-420, 2005.

18. Bridges JR and Cecil C: Hierarchical cluster analysis. Psycho Rep 18: 851-854, 1966

19. Read T, Vance D and BluePrints S: Robust Clustering: A Comparison of Sun $^{\mathrm{TM}}$ Cluster 3.0 versus Sun Cluster 2.2 Software. Sun BluePrints ${ }^{\mathrm{TM}}$ OnLine 2001: 1-23, 2001.

20. Zhai Y, Tchieu J and Saier MH Jr: A web-based Tree View (TV) program for the visualization of phylogenetic trees. J Mol Microbiol Biotechnol 4: 69-70, 2002.
21. Di Pietro C, Di Pietro V, Emmanuele G, Ferro A, Maugeri T, Modica E, Pigola G, Pulvirenti A, Purrello M, Scalia M, et al: Anticlustal: Multiple sequence alignment by antipole clustering and linear approximate 1-median computation. Proc IEEE Comput Soc Bioinform Conf 2: 326-336, 2003.

22. Chen X: Curve-based clustering of time course gene expression data using self-organizing maps. J Bioinform Comput Biol 7: 645-661, 2009.

23. Ashburner M, Ball CA, Blake JA, Botstein D, Butler H, Cherry JM, Davis AP, Dolinski K, Dwight SS, Eppig JT, et al: Gene ontology: Tool for the unification of biology. The gene ontology consortium. Nat Genet 25: 25-29, 2000.

24. Kanehisa M and Goto S: KEGG: Kyoto encyclopedia of genes and genomes. Nucleic Acids Res 28: 27-30, 2000.

25. Huang DW, Sherman BT, Tan Q, Kir J, Liu D, Bryant D, Guo Y, Stephens R, Baseler MW, Lane HC and Lempicki RA: DAVID Bioinformatics resources: Expanded annotation database and novel algorithms to better extract biology from large gene lists. Nucleic Acids Res 35: W169-W175, 2007.

26. Krishnapuram B, Carin L and Hartemink A: 1 Gene expression analysis: Joint feature selection and classifier design. In: Kernel Methods in Computational Biology. Schölkopf B, Tsuda K and Vert JP (eds). MIT Press, Cambridge, pp299-317, 2004.

27. Liu T, Tao P, Li X, Qin Y and Wang C: Prediction of subcellular location of apoptosis proteins combining tri-gram encoding based on PSSM and recursive feature elimination. J Theor Biol 366: 8-12, 2015

28. Ruiz MJ, Hupé JM and Dojat M: Use of pattern-information analysis in vision science: A pragmatic examination. In: Machine learning in medical imaging. Suzuki K, Yan P, Wang F and Shen D (eds). Springer, New York, pp103-110, 2012.

29. Ma S, Lv M, Deng F, Zhang X, Zhai H and Lv W: Predicting the ecotoxicity of ionic liquids towards Vibrio fischeri using genetic function approximation and least squares support vector machine. J Hazard Mater 283: 591-598, 2015.

30. Pedregosa F, Varoquaux G, Gramfort A, Michel V, Thirion B, Grisel O, Blondel M, Prettenhofer P, Weiss R, Dubourg V and Vanderplas J: Scikit-learn: Machine learning in Python. J Mach Learn Res 12: 2825-2830, 2011.

31. Shah PK: Biomarkers of plaque instability. Curr Cardiol Rep 16: 547,2014

32. Beel AJ and Sanders CR: Substrate specificity of gamma-secretase and other intramembrane proteases. Cell Mol Life Sci 65: 1311-1334, 2008

33. Liu ZJ, Tan Y, Beecham GW, Seo DM, Tian R, Li Y, Vazquez-Padron RI, Pericak-Vance M, Vance JM, Goldschmidt-Clermont PJ, et al: Notch activation induces endothelial cell senescence and pro-inflammatory response: Implication of Notch signaling in atherosclerosis. Atherosclerosis 225: 296-303, 2012.

34. Fukuda D, Aikawa E, Swirski FK, Novobrantseva TI, Kotelianski V, Gorgun CZ, Chudnovskiy A, Yamazaki H, Croce K, Weissleder R, et al: Notch ligand delta-like 4 blockade attenuates atherosclerosis and metabolic disorders. Proc Natl Acad Sci USA 109: E1868-E1877, 2012.

35. Casserly I and Topol E: Convergence of atherosclerosis and Alzheimer's disease: Inflammation, cholesterol, and misfolded proteins. Lancet 363: 1139-1146, 2004.

36. van Loo KM, Dejaegere T, van Zweeden M, van Schijndel JE, Wijmenga C, Trip MD and Martens GJ: Male-specific association between a gamma-secretase polymorphism and premature coronary atherosclerosis. PloS One 3: e3662, 2008.

37. Santoso S, Sachs UJ, Kroll H, Linder M, Ruf A, Preissner KT and Chavakis T: The junctional adhesion molecule 3 (JAM-3) on human platelets is a counterreceptor for the leukocyte integrin Mac-1. J Exp Med 196: 679-691, 2002.

38. Ling S, Nheu L and Komesaroff PA: Cell adhesion molecules as pharmaceutical target in atherosclerosis. Mini Rev Med Chem 12: 175-183, 2012.

39. Galkina E and Ley K: Vascular adhesion molecules in atherosclerosis. Arterioscler Thromb Vasc Biol 27: 2292-2301, 2007.

40. Shagdarsuren E,Djalali-Talab Y,Aurrand-Lions M, et al: Importance of Junctional adhesion molecule-C for neointimal hyperplasia and monocyte recruitment in atherosclerosis-prone mice-brief report. Arterioscler Thromb Vasc Biol 29: 1161-1163, 2009.

41. Skov V, Knudsen S, Olesen M, Hansen ML and Rasmussen LM: Global gene expression profiling displays a network of dysregulated genes in non-atherosclerotic arterial tissue from patients with type 2 diabetes. Cardiovasc Diabetol 11: 15, 2012. 
42. Lin-Tsai O, Clark PE, Miller NL, et al: Surgical intervention for symptomatic benign prostatic hyperplasia is correlated with expression of the AP-1 transcription factor network. Prostate 74: 669-679, 2014

43. Chang L, Xu J, Yu F, Zhao J, Tang X and Tang C: Taurine protected myocardial mitochondria injury induced by hyperhomocysteinemia in rats. Amino Acids 27: 37-48, 2004.

44. Wójcik OP, Koenig KL, Zeleniuch-Jacquotte A, Costa M and Chen Y: The potential protective effects of taurine on coronary heart disease. Atherosclerosis 208: 19-25, 2010.
45. Grosse J, Chitu V, Marquardt A, Hanke P, Schmittwolf C, Zeitlmann L, Schropp P, Barth B, Yu P, Paffenholz R, et al: Mutation of mouse Mayp/Pstpip2 causes a macrophage autoinflammatory disease. Blood 107: 3350-3358, 2006.

46. Pixley FJ and Stanley ER: CSF-1 regulation of the wandering macrophage: Complexity in action. Trends Cell Biol 14: 628-638, 2004.

47. Chitu V and Stanley ER: Colony-stimulating factor-1 in immunity and inflammation. Curr Opin Immunol 18: 39-48, 2006. 